\title{
Novos táxons e notas taxonômicas em Prioninae (Coleoptera, Cerambycidae)
}

\author{
Antonio Santos-Silva ${ }^{1} \&$ Ubirajara R. Martins ${ }^{1,2}$ \\ 1. Museu de Zoologia, Universidade de São Paulo, Caixa Postal 42494, 04299-970 São Paulo, SP, Brasil. \\ 2. Pesquisador do CNPq.
}

\begin{abstract}
New taxa and taxonomic notes in Prioninae (Coleoptera, Cerambycidae). Hisarai, a new genus of Macrotomini, is described to allocate H. seripierriae (Santos-Silva \& Martins, 2003), new combination, originally described in Physopleurus Lacordaire, 1869. New species described from Brazil (Bahia): Nothopleurus komiyai (Macrotomini). New synonymy in Macrotomini: Mallodon baroni Casey, 1912 = Mallodon dasystomus dasystomus (Say, 1824). New status in Callipogonini: Spiloprionus Aurivillius, 1897 and Navosoma Blanchard, 1846 (with discussion on dates of Navosoma and N. triste Blanchard, 1846). Key to the genera of American Macrotomini is added.
\end{abstract}

KEYWORDS. Callipogonini, Hisarai, Macrotomini, new taxa, taxonomy.

RESUMO. Hisarai, novo gênero de Macrotomini, é descrito para alocar H. seripierriae (Santos-Silva \& Martins, 2003), nova combinação, originalmente descrito em Physopleurus Lacordaire, 1869. Nova espécie descrita do Brasil (Bahia): Nothopleurus komiyai (Macrotomini). Nova sinonímia em Macrotomini: Mallodon baroni Casey, 1912 = Mallodon dasystomus dasystomus (Say, 1824). Novos status em Callipogonini: Spiloprionus Aurivillius, 1897 e Navosoma Blanchard, 1846 (com discussão sobre as datas de Navosoma e N. triste Blanchard, 1846). Uma chave para os gêneros Americanos de Macrotomini é adicionada.

PALAVRAS-CHAVE. Callipogonini, Hisarai, Macrotomini, novos táxons, taxonomia.

A dificuldade para caracterizar os gêneros de Macrotomini faz com que, muitas vezes, as descrições genéricas sejam muito restritas ou, ao contrário, muito amplas. A descoberta de espécies novas, freqüentemente, torna necessário rever conceitos genéricos estabelecidos e, ao mesmo tempo, preenchem lacunas entre espécies alocadas em gêneros diferentes. Essas espécies intermediárias entre dois gêneros tornam ainda mais difícil estabelecer seus limites. A descrição de Hisarai gen. nov. eleva para 18 o número de gêneros de Macrotomini americanos, entre os quais, sete monotípicos: Mallodonhoplus Thomson, 1860; Ialyssus Thomson, 1864; Hisarai gen. nov.; Basitoxus Audinet-Serville, 1832; Protorma Waterhouse, 1880; Chiasmetes Pascoe, $1867 \mathrm{e}$ Neomallodon Linsley, 1957.

As citações bibliográficas sob Mallodon dasystomus dasystomus (Say, 1824), relativas à sinonímia proposta, estão restritas às descrições originais (inclusive sinônimos), ao catálogo de MonNÉ (1995), às omissões e citações subseqüentes; aquelas sob Navosoma não incluem os nomencladores zoológicos, exceto os relativos aos nomes inválidos.

Navosoma Blanchard, 1846 e Spiloprionus Aurivillius, 1897, atualmente considerados subgêneros de Callipogon Audinet-Serville, 1832, são elevados a gênero, por possuírem caracteres que permitem sua separação.

As siglas utilizadas correspondem às seguintes instituições: INPA, Instituto Nacional de Pesquisas da Amazônia, Manaus; MNRJ, Museu Nacional, Universidade Federal do Rio de Janeiro, Rio de Janeiro; MZSP, Museu de Zoologia, Universidade de São Paulo, São Paulo; USNM, National Museum of Natural History, Washington D.C.

\section{Macrotomini Hisarai gen. nov.}

Etimologia. O gênero é dedicado a Hisayasu Arai, pesquisador e colecionador japonês, pela doação de espécimes ao MZSP. Gênero masculino.

Espécie-tipo. Physopleurus seripierriae SantosSilva \& Martins, 2003.

Macho (Fig. 1). Tegumento castanho. Largura da cabeça (Fig. 3) igual a aproximadamente $2 / 3$ da largura dos élitros na base; região dorsal fortemente pontuada. Suturas frontais e coronal nítidas em toda extensão, ou as frontais pouco distintas. Sutura epistomal ausente. Ápice dos tubérculos anteníferos saliente, arredondado e inclinado em direção à lateral. Base do labro coplanar com o clípeo. Mento fortemente transverso. Ápice das genas nitidamente projetado e aguçado. Olhos mais longos do que largos; lobos oculares superiores (Fig. 3) mais afastados que o quádruplo da largura de um lobo. Lígula inteira ou com reentrância suave na borda anterior. Palpos maxilares alongados; segundo artículo mais longo do que o terceiro. Mandíbulas (Fig. 3) aproximadamente tão longas quanto a cabeça, falciformes; carena dorsal larga e elevada na base; margem interna com único dente, aguçado, próximo do ápice. Antenas apenas ultrapassam o meio dos élitros; escapo (Fig. 3) ultrapassa a borda posterior dos olhos, subigual em comprimento aos antenômeros III-IV reunidos. Protórax (Fig. 3) transversal, com ângulos anteriores salientes; margens laterais fortemente crenadas. Pronoto com pontuação grossa e cerrada, exceto no disco, onde há áreas lisas. Prosterno 
convexo, fraca e esparsamente pontuado. Suturas prosternais (Fig. 4) presentes e apenas curvas. Esternos torácicos pubescentes. Élitros glabros, rugosos na base e com pontos grossos e rasos no restante; ápice com espinho sutural. Fêmures com espinhos curtos na face ventral, mais numerosos nos profêmures. Tíbias nitidamente espinhosas nas faces laterais.

Fêmea (Fig. 2). Distância entre os lobos oculares superiores igual a aproximadamente o triplo da largura de um lobo. Mandíbulas semelhantes às dos machos, porém mais curtas; margem interna com ou sem dente mediano. Escapo não alcança a borda posterior dos olhos. Antenas não atingem o meio dos élitros.

Discussão. Santos-Silva \& Martins (2003) descreveram Physopleurus seripierriae baseados em três fêmeas e comentaram: "É a espécie cujas suturas prosternais apresentam menor curvatura em direção ao pronoto (provavelmente as suturas do macho são mais curvadas)". O exame de dois machos mostra que as suturas prosternais são menos curvas do que supúnhamos e esse caráter, associado aos listados abaixo, permitiu erigir um novo gênero para essa espécie.

Hisarai gen. nov. difere de Physopleurus Lacordaire, 1869 (principalmente nos machos): tubérculos anteníferos não-aguçados; mandíbulas falciformes, com comprimento igual a aproximadamente o triplo da largura no centro e com a margem interna pouco saliente; prosterno apenas convexo; suturas prosternais pouco curvadas em direção ao pronoto a partir da base das procoxas; metatíbias nitidamente espinhosas. Em Physopleurus, os tubérculos anteníferos, em geral, são aguçados; as mandíbulas não são falciformes, seu comprimento é aproximadamente igual ao dobro da largura no centro (exceto em P. tritomicros Lameere, 1912) e a margem interna é saliente; o prosterno é freqüentemente intumescido; as suturas prosternais são nitidamente curvadas em direção ao pronoto a partir da base das procoxas; as metatíbias são inermes ou têm espinhos curtos e muito esparsos.

Difere de Ialyssus Thomson, 1864, pela cabeça visivelmente maior e mais larga (Figs. 1-3), pelas mandíbulas (Figs. 1-3) mais longas (principalmente nos machos), pela ausência de dimorfismo sexual no pronoto e pelos tarsos (Figs. 5, 6) mais estreitos (principalmente os anteriores). Em Ialyssus ver Figs. 10-13.

Difere ainda de Mallodonopsis Thomson, 1860, pelo comprimento do escapo subigual aos antenômeros III-IV reunidos; pelo labro coplanar com o clípeo; pelas mandíbulas mais estreitas (Fig. 3); pela sutura prosternal curvada e pelas margens do protórax fortemente crenadas. Em Mallodonopsis (Fig. 14), o escapo é mais longo que os antenômeros III-IV reunidos, o labro não é coplanar com a borda do clípeo, as mandíbulas são mais largas (Fig. 14), a sutura prosternal é sub-reta e as margens do protórax são espinhosas.

\section{Hisarai seripierriae (Santos-Silva \& Martins, 2003) comb.nov.}

(Figs. 1-6)

Physopleurus seripierriae SAntos-Silva \& Martins, 2003: 255, figs. 66-71.
Macho (Figs. 1, 3-6). A descrição da fêmea (Fig. 2), associada aos caracteres listados na descrição do gênero e as fotografias inseridas, permitem reconhecer facilmente os machos dessa espécie.

Material examinado (além dos tipos de Physopleurus seripierriae). BRASIL. Amazonas: Manaus, O’, XI.1996, [sem nome do coletor] (MZSP, doação de Z. Komiya); Estrada ZF-2

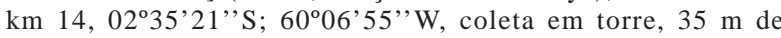
altitude, com luz mista e BLB, O', 13-16.IX.2004, F. F. Xavier Fo A. R. Ururahy, F. Godoy \& T. Trovisco col. (INPA); $75 \mathrm{~km} \mathrm{~S}$ Manaus, ơ, 8.II.1993, H. Arai col. (MZSP, doação de H. Arai).

\section{Nothopleurus komiyai sp. nov.}

(Figs. 7-9)

Etimologia. Espécie dedicada a Ziro Komiya, pesquisador japonês, pela doação do holótipo à coleção do MZSP.

Fêmea (Fig. 7). Tegumento castanho; cabeça, mandíbulas, antenas e laterais do pronoto, castanhoescuro.

Região dorsal da cabeça rugosa junto à carena ocular, grossa e cerradamente pontuada em direção ao occipício; área entre os lobos oculares com gibosidade brilhante, esparsa e grossamente pontuada; carena ocular estreita e elevada; pilosidade esparsa na gibosidade e relativamente abundante junto aos olhos e laterais da cabeça. Sutura coronal marcada até o nível da borda posterior dos olhos. Distância entre os lobos oculares superiores igual ao triplo da largura de um lobo. Fronte com pêlos longos e abundantes. Genas separadas da região hipostomal por carena saliente, elevada em direção às mandíbulas; pontuação grossa e abundante; pilosidade relativamente abundante; ápice saliente. Área hipostomal (Fig. 8) grosseiramente vermiculada, com pêlos longos e abundantes. Artículo II dos palpos maxilares e labiais mais longo do que os demais. Gálea atinge o terço basal do artículo II dos palpos maxilares. Lígula pequena, ápice das paraglossas aguçado. Mandíbulas 1/3 mais curtas do que a cabeça; carena dorsal estreita e elevada nos dois terços basais (principalmente no centro dessa região), onde termina; face interna com pontos grossos e pilosidade abundante, ambos mais esparsos em direção ao ápice; margem interna irregularmente crenulada, com dente pequeno junto ao ápice. Antenas atingem apenas o terço basal dos élitros; escapo alcança o meio dos olhos, alargado em direção ao ápice; antenômero III (Fig. 9) subigual, em comprimento, ao escapo e com quase o dobro do comprimento do antenômero IV.

Protórax transversal com espinhos nítidos nas bordas laterais, mais estreito do que os élitros; ângulos anteriores não-projetados para frente; ângulos posteriores indicados. Borda anterior do pronoto côncava; disco com calosidades brilhantes, fracamente separadas por pontos grossos e cerrados e unidas à uma gibosidade transversal junto à borda posterior; laterais com pontos muito grossos e anastomosados; pilosidade esparsa nas laterais. Partes laterais do metasterno e metepisternos com pilosidade curta e abundante. Metepisternos nitidamente côncavos no centro. Élitros lisos e brilhantes, ápice com espinho sutural curto. Urosternitos brilhantes, com pêlos 


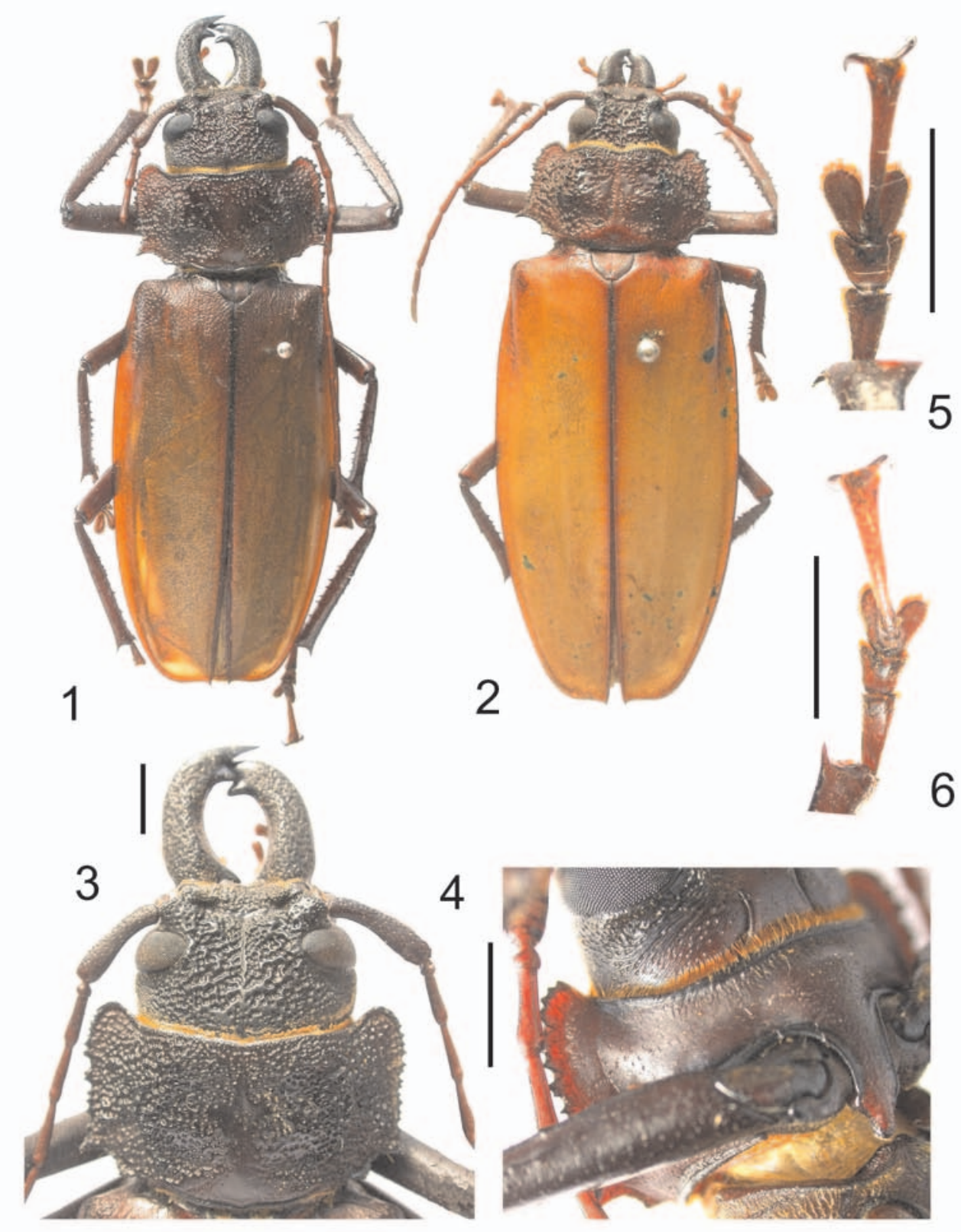

Figs. 1-6. Hisarai seripierriae (Santos-Silva \& Martins, 2003): 1, Ođ, vista dorsal, comprimento 64,0 mm; 2, parátipo + , vista dorsal,

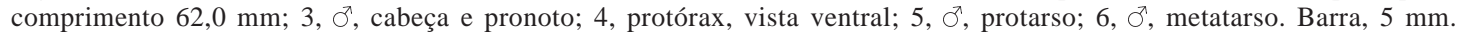

esparsos e muito curtos na região central e mais longos nas laterais; pontuação muito fina e esparsa; ápice do último urosternito truncado. Tíbias com pontos grossos e relativamente abundantes, principalmente as protíbias.

Dimensões em mm, fêmea. Comprimento total, 41,0; comprimento do protórax no centro, 6,0; largura do protórax entre os ângulos anteriores, 9,2; largura do protórax entre os ângulos laterais, 11,3; largura do protórax entre os ângulos posteriores, 9,3; largura umeral, 12,2; comprimento elitral, 26,0.

Material-tipo. BRASIL, Bahia: Santo Antonio, holótipo
+ , XI.1950, [sem nome do coletor], (MZSP, doação de Ziro Komiya). Existem numerosas "Santo Antonio" nesse Estado (CALle.com, 2004).

Discussão. A localidade-tipo de Nothopleurus komiyai sp. nov. é duvidosa porque a espécie possui caracteres próprios das espécies da América Central, principalmente a presença de carena nítida entre as genas e a área hipostomal.

A nova espécie é semelhante à $N$. lobigenis Bates, 1884 (Fig. 15), mas difere (fêmea): comprimento do escapo (Fig. 9) igual a 2/3 da distância entre os lobos oculares superiores e antenômero III bem mais longo do que o IV; 

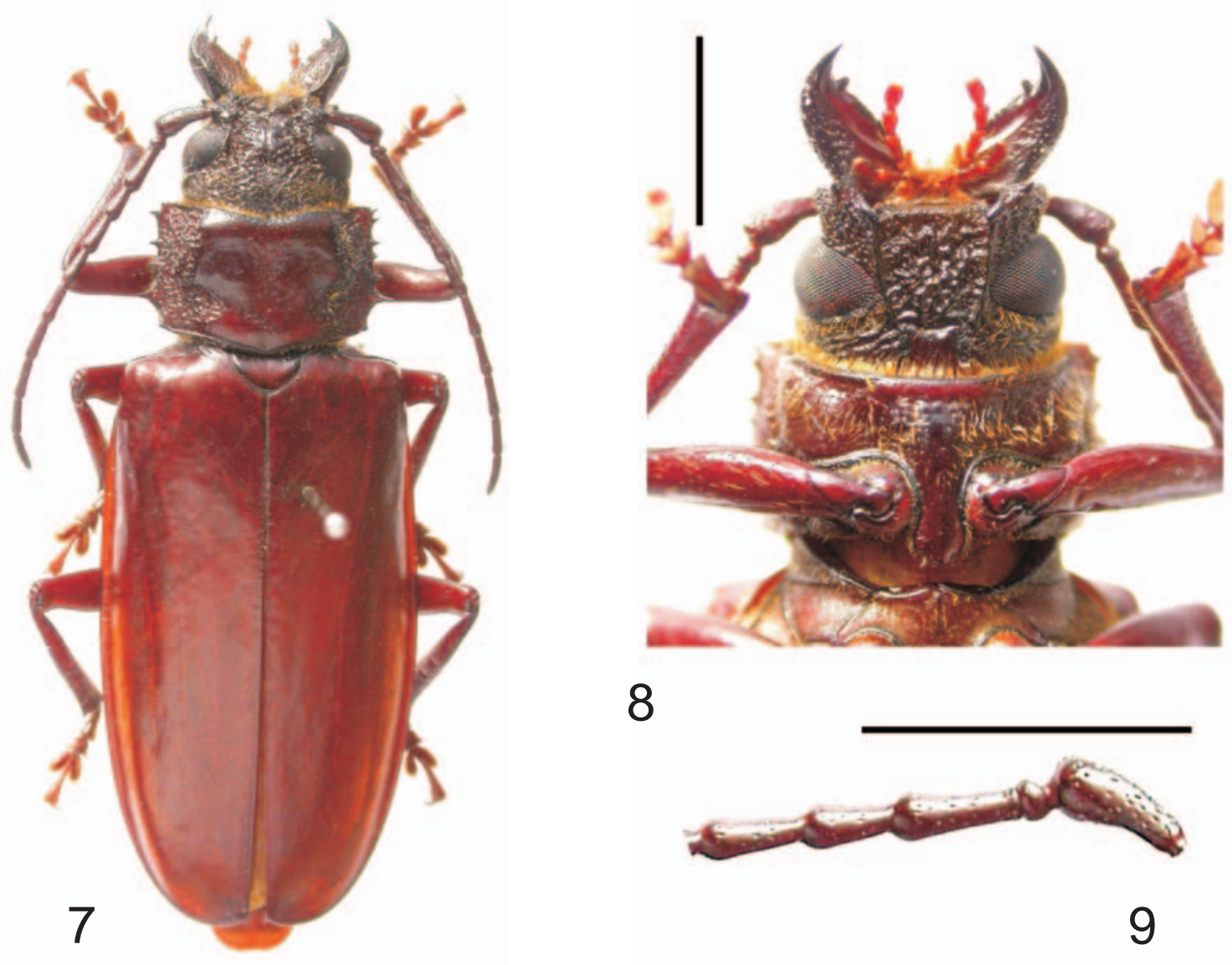

8

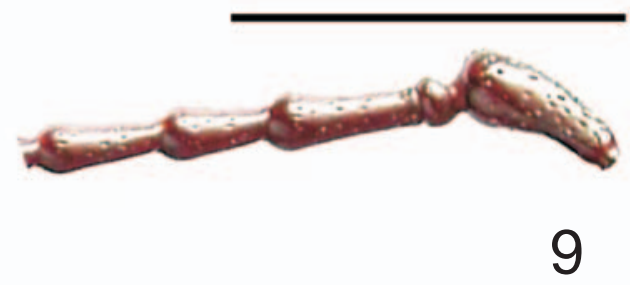

Figs. 7-9. Nothopleurus komiyai sp. nov., holótipo $9:$, vista dorsal, comprimento 41,0 mm; 8, cabeça e protórax, vista ventral; 9 , antena, segmentos basais. Barra, $5 \mathrm{~mm}$.

protórax (Fig. 7) 1/3 mais largo do que longo e com as margens laterais nitidamente espinhosas. Em N. lobigenis (fêmea) o comprimento do escapo é igual à metade da distância entre os lobos oculares superiores, o antenômero III é apenas mais longo do que o IV, a largura do protórax é quase o dobro do seu comprimento e as margens laterais são fracamente espinhosas. Diferenciase de $N$. bituberculatus (Beauvois, 1805), pelo escapo apenas curvo, pelo antenômero III mais longo do que o IV, pelas mandíbulas aproximadamente tão longas quanto a distância entre os lobos oculares superiores, pela carena entre a gena e área hipostomal mais elevada, pela área hipostomal menor e pelo protórax com margens laterais subparalelas, com espinhos finos. Em $N$. bituberculatus (fêmea) (Fig. 18), o escapo é curvo, o comprimento do antenômero III é subigual ao do IV, as mandíbulas são menores do que a distância entre os lobos oculares superiores, a carena entre a gena e a área hipostomal é mais baixa, a área hipostomal é maior e as margens laterais do protórax são crenuladas e não subparalelas. Nas fêmeas de N. maxillosus (Drury, 1773) [Fig. 19 - baseado em VILLIERS (1980: 141)] o escapo é mais estreito no ápice e as margens laterais do pronoto são convergentes para frente. Diferencia-se da fêmea de N. madericus (Skiles,
1978), principalmente pelas mandíbulas mais retas na face externa e pela área hipostomal mais longa e bem delimitada pelas carenas laterais. As fêmeas de $N$. madericus possuem mandíbulas nitidamente curvadas na face externa e a área hipostomal mais curta e irregularmente delimitada pelas carenas laterais. Não examinamos nenhuma fêmea de $N$. subsulcatus (Dalman, 1823), mas a fotografia do holótipo macho (além do holótipo macho de Mallodon gnatho White, 1853 - sinônimo), permite inferir que a mandíbula da fêmea é diferente. Difere da fêmea de $N$. subcancellatus (Thomson, 1867) pelas mandíbulas mais longas (Fig. 7), pela forma e comprimento do escapo e antenômero III (Fig. 9), pela presença de carena nítida entre a gena e a área hipostomal e pela forma geral do protórax (Fig. 7). Nas fêmeas de N. subcancellatus (Fig. 16) as mandíbulas são mais curtas, o escapo é curvo, o comprimento do antenômero III é subigual ao do IV, as genas não são separadas da área hipostomal por carena, as margens laterais do protórax são crenuladas e os ângulos anteriores são projetados para frente. De $N$. santacruzensis Hovore \& Santos-Silva, 2004, difere pelas mandíbulas mais longas, pela distância entre os lobos oculares superiores (Fig. 7) igual ao triplo da largura de um lobo, pelo escapo apenas mais longo do que o 


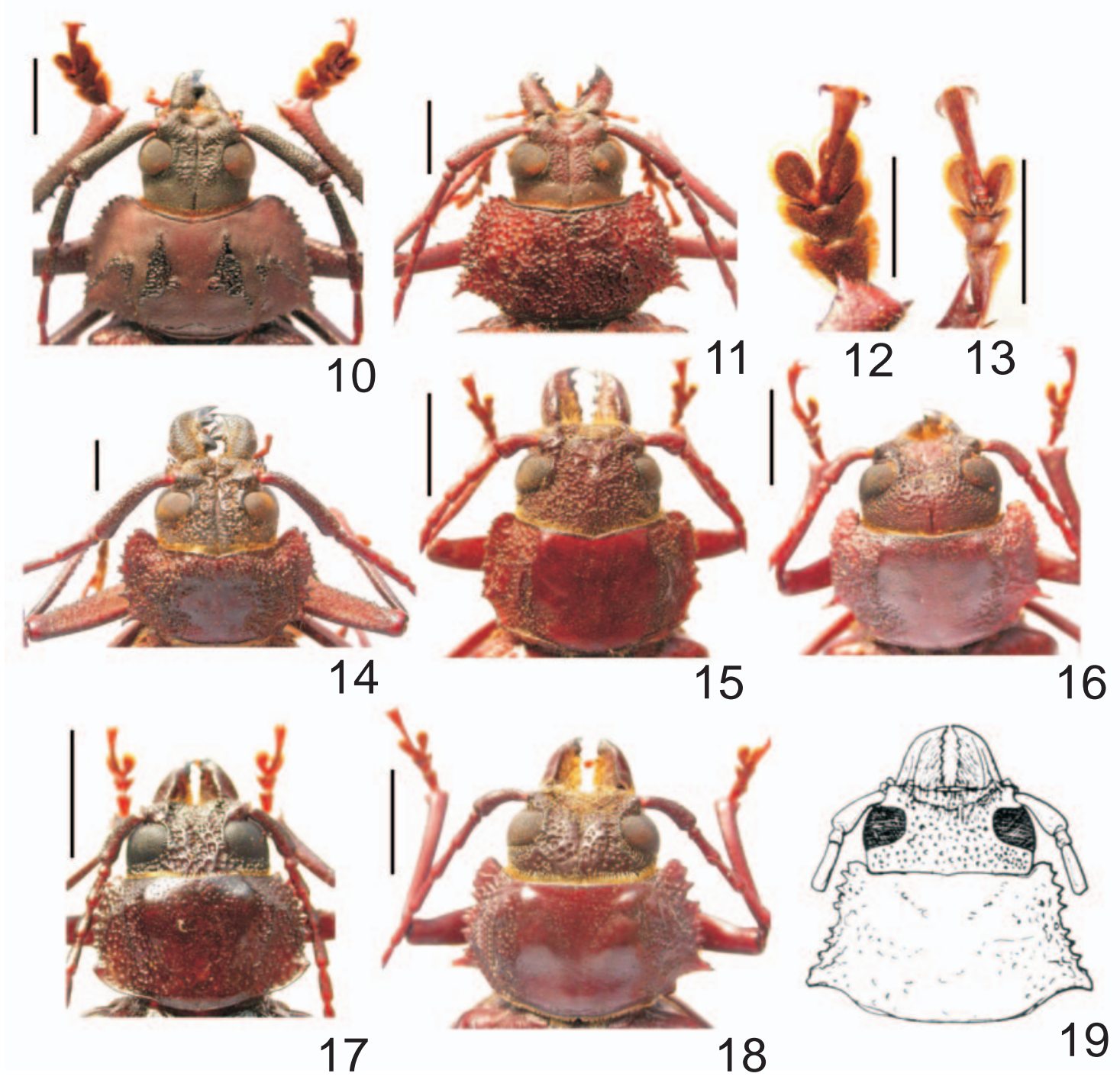

Figs. 10-19. 10-13, Ialyssus tuberculatus (Olivier, 1795); 10, $\sigma^{2}$, cabeça e pronoto; 11, $\odot$, cabeça e pronoto; 12, $\sigma^{2}$, protarso; 13, $\sigma^{2}$,

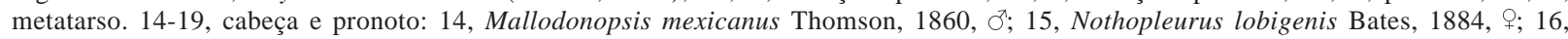
N. subcancellatus (Thomson, 1867), ; 17, N. santacruzensis, parátipo $\odot ; 18, N$. bituberculatus (Beauvois, 1805), ; 19, N. maxilosus, (Drury, 1773), ㅇ [VILLIERS (1980): fig. 7]. Barra, $5 \mathrm{~mm}$.

antenômero III, pela carena nítida entre a gena e a área hipostomal e pela forma geral do protórax. Em $N$. santacruzensis fêmea (Fig. 17), as mandíbulas são mais curtas, a distância entre os lobos oculares superiores é igual ao dobro da largura de um lobo, o escapo é quase duas vezes mais longo do que o antenômero III, as bordas laterais do protórax são curvas e crenuladas e a carena que separa a gena da área hipostomal é muito baixa.

\section{Mallodon dasystomus dasystomus (Say, 1824) (Figs. 20-24)}

Prionus dasystomus SAY, 1824:326.

Mallodon costulata LECONTE, 1851:111.

Mallodon dasystoma LeConte, 1851:112.

Mallodon masticator Thomson, 1867:99.

Mallodon angustatum Тномsоn, 1867:100.

Mallodon degeneratum Thомson, 1867:95.

Mallodon debile CASEY, 1912:222.

Stenodontes (Orthomallodon) dasytomus dasytomus [sic]; NoguerA
\& CHEMSAK, 1996:396 (distr.).

Stenodontes (Orthomallodon) dasytomus masticator; NOGUERA \& CHEMSAK, 1996: 396 (distr.).

Stenodontes dasytomus [sic]; Browne \& Peck, 1996:2158, 2160 (distr.); Chemsak \& Noguera, 1993:57; Toledo et al., 2002: 525 (distr.).

Mallodon dasystomus; CHEMSAK, 1996:78, pl. IV, figs. 11-12 (syn.); MonNé, 2002:13 (hosp.).

Mallodon dasystomus dasystomus; Monné, 1995:9 (cat.); Hovore \& SANTOS-Silva, 2004:51.

Mallodon dasytomus dasytomus [sic]; Yanega, 1996:25, pl. 1, figs. 6a-6b; LinsLey \& CHEMSAK, 1997:434 (hosp.).

Mallodon mandibularis LACKERBECK, 1998:517 [non Gemminger, 1872]; Hovore \& Santos-Silva, 2004:51 (syn).

Mallodon baroni CASey, 1912:223; Linsley, 1957:4. Syn. nov.

Mallodon Baroni; LAMEERE, 1919: 32 (syn).

Stenodontes (Orthomallodon) baroni; CHEMSAK \& LinsLey, 1982:2 (cat.; reval.); ChemsaK et al., 1992:15 (cat.); Noguera \& CHEMSAK, 1996:396 (distr.).

Stenodontes (Mallodon) baroni; Monné \& GIESBERT, 1994:6 (cat.). Nothopleurus baroni; Fragoso \& MonNé, 1995:219.

Nothopleurus baroni; MonNé, 1995:13 (cat.). 

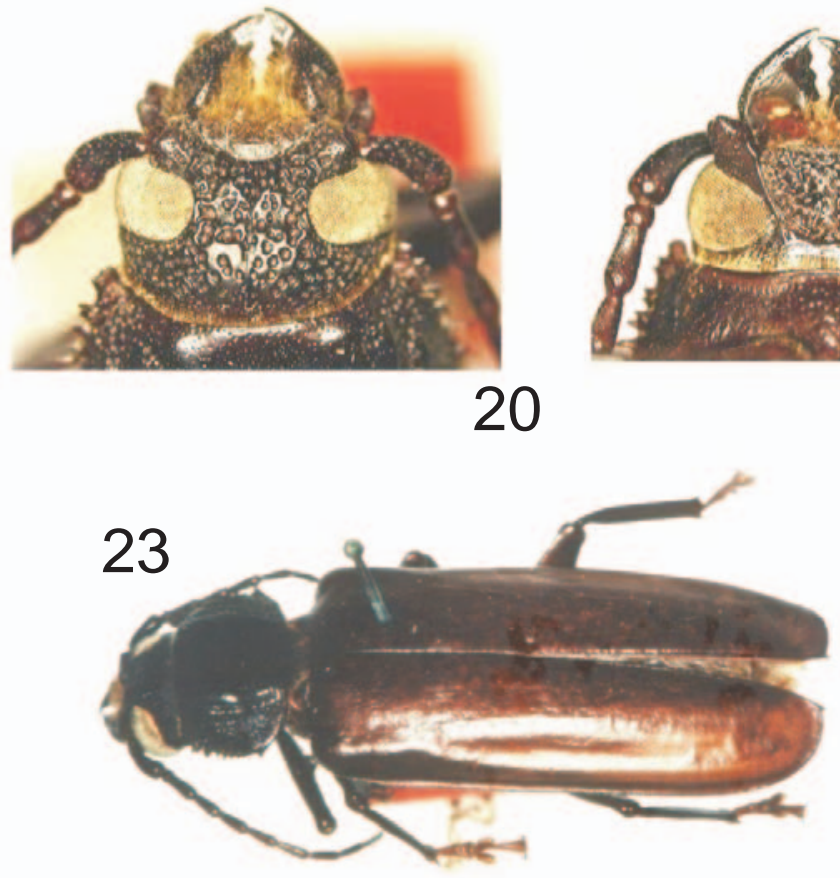
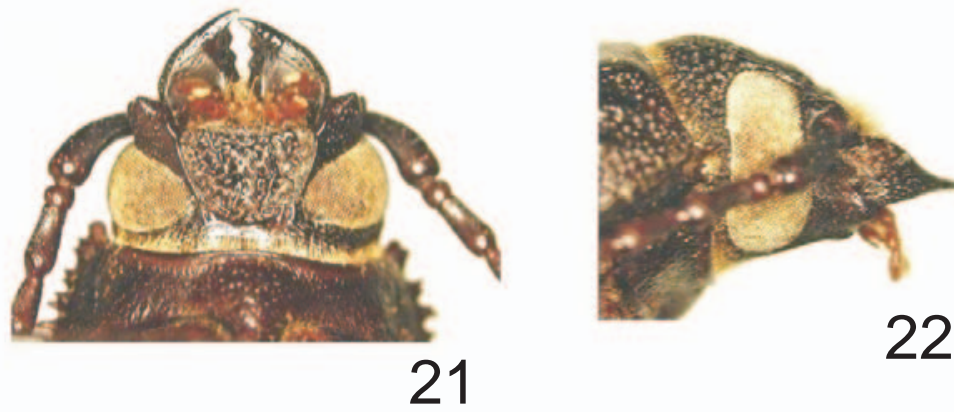

22

Figs. 20-24. Mallodon baroni Casey, 1912, holótipo P: 20, cabeça, vista dorsal; 21, cabeça, vista ventral; 22, cabeça, vista lateral; 23 , corpo, vista dorsal, comprimento $35,0 \mathrm{~mm} ; 24$, metepisterno.

LAMEere (1919) colocou Mallodon baroni Casey, 1912 (Fig. 23), com dúvida, na sinonímia de Mallodon spinibarbis (Linnaeus, 1758). LinsLEY (1957), escreveu: "Mallodon baroni Casey (from Guerrero, Mexico), placed questionably as a synonym of Stenodontes spinibarbis Linnaeus by Lameere, has the genae more or less tridentate as in S. dasystomus masticator (Thomson) and may be that subspecies". Posteriormente, CHEMSAK \& LiNSLEY (1982), revalidaram a espécie como Stenodontes (Orthomallodon) baroni. MonNÉ \& GiesBerT (1994:6, cat.) citaram como Stenodontes (Mallodon) baroni. FRAGOSO \& MonNé (1995) transferiram a espécie para o gênero Nothopleurus Lacordaire, 1869. O exame das fotografias enviadas por Steven W. Lingafelter (USNM), permitiu concluir que LiNSLEY (1957) estava certo ao supor a sinonímia com Mallodon masticator [= Mallodon dasystomus, de acordo com CHEMSAK (1996)]. Embora seja questionável a sinonímia de $M$. masticator (tipo não examinado) com $M . d$. dasystomus, a sinonímia com essa última é, em nossa opinião, bastante clara. O metepisterno (Fig. 24) é nitidamente largo e com a borda interna convexa, característica que a exclui do gênero Nothopleurus, no qual esse esclerito é estreito e, em geral, com a borda interna côncava. A forma e escultura da cabeça (Figs. 20-22), particularmente a gena, permitiu estabelecer a sinonímia.

Chave para os gêneros americanos de Macrotomini

1. Escutelo nitidamente convexo e áspero
Escutelo plano ou deprimido e sem asperezas .. 5

2(1). Antenas serradas

Parastrongylaspis Giesbert, 1987

Antenas filiformes

3(2). Escutelo sem sulco longitudinal ou com sulco restrito à base

Strongylaspis Thomson, 1860

Escutelo dividido por sulco longitudinal da base ao ápice

... 4

4(3). Escapo atinge ou ultrapassa a borda posterior dos olhos; élitros com pontuação grossa em toda superfície. ...Curitiba Lameere, 1903

Escapo não atinge a borda posterior dos olhos; élitros rugosos ou ásperos no terço basal e com pontuação moderadamente fina no restante .

Chiasmetes Pascoe, 1867

5(1). Escutelo deprimido no disco e com margens laterais elevadas e estreitas

Protorma Waterhouse, 1880

Escutelo não deprimido no disco ou deprimido na área centro-anterior, as margens laterais nunca elevadas e estreitas ......................................... 6

6(5). Mandíbulas subverticais (face inferior das mandíbulas forma ângulo menor que $150^{\circ} \mathrm{com}$ a superfície ventral do corpo) 7

Mandíbulas horizontais ou suborizontais (face inferior das mandíbulas forma ângulo maior que $150^{\circ}$ com a superfície ventral do corpo) (........ 8 .2
7(6). Escapo ultrapassa a borda posterior dos olhos; 
antenas nos machos, excedem o meio dos élitros e nas fêmeas, atingem o terço basal ... Mallodonopsis Thomson, 1860

Escapo não atinge ou apenas atinge a borda posterior dos olhos; antenas nos machos, alcançam apenas o terço basal dos élitros e nas fêmeas, o quarto basal

Archodontes Lameere, 1903

8(6). Metepisterno estreito (maior largura igual a aproximadamente $1 / 4$ do comprimento), com a borda interna freqüentemente côncava ......... Nothopleurus Lacordaire, 1869

Metepisterno largo (maior largura igual a aproximadamente $1 / 3$ do comprimento), com a borda interna reta ou fracamente convexa.... 9

9(8). Margens laterais do protórax com espinhos longos ........ Aplagiognathus Thomson, 1860

Margens laterais do protórax lisas, ou crenuladas, ou com espículos

10(9). Metatíbias com espinhos numerosos (se houver espinhos nítidos e o escapo for, no minímo, duas vezes mais longo do que o antenômero III, vide dilema 12 - Mecosarthron) ............ 11

Metatíbias inermes ou no máximo com alguns espinhos

11(10).Cabeça aproximadamente tão larga quanto o comprimento do escapo; escultura do pronoto dos machos diferente daquela das fêmeas .... Ialyssus Thomson, 1864

Cabeça claramente mais larga do que o comprimento do escapo; pronoto com escultura semelhante em ambos os sexos

Hisarai gen. nov.

12(10). Élitros pubescentes

Mecosarthron Buquet, 1840

Élitros glabros 13

13(12). Sutura prosternal curva ou ausente; proepisterno dos machos freqüientemente reduzido ....... 14

Sutura prosternal reta; proespisterno evidente nos dois sexos 15

14(13). Tubérculos anteníferos suborizontais; margem látero-externa das mandíbulas arredondada ..

Physopleurus Lacordaire, 1869

Tubérculos anteníferos eretos; margem láteroexterna das mandíbulas sub-reta

Mallodonhoplus Thomson, 1860

15(13). Mandíbulas mais curtas do que a cabeça nos dois sexos e com a face látero-externa nitidamente intumescida

Neomallodon Linsley, 1957

Mandíbulas em geral mais longas do que a cabeça (principalmente nos machos), com a face láteroexterna não intumescida [exceto em Mallodon popelairei (Lameere, 1902)]

.. 16

16(15). Antenas ultrapassam o meio dos élitros nos machos (freqüientemente atingem o terço apical) e atingem o meio dos élitros nas fêmeas ........

Stenodontes Audinet-Serville, 1832

Antenas atingem no máximo o meio dos élitros nos machos e o terço basal nas fêmeas ...... 17

17(16). Protórax nitidamente transversal nos dois sexos, sem espinho longo no terço látero-posterior e com a borda oblíqua desse ponto até a base; élitros da mesma cor que o restante do corpo ou suavemente mais claros

Mallodon Lepeletier \& Audinet-Serville, 1830

Protórax apenas transversal nos dois sexos, com espinho longo no terço látero-posterior e com reentrância curva, acentuada, atrás desse espinho; élitros nitidamente mais claros do que o restante do corpo Basitoxus Audinet-Serville, 1832

\section{Callipogonini}

\section{Navosoma Blanchard, 1846 stat. nov.}

Navosoma AgAssiz, 1846a:106 (nomen nudum)

Navosoma AgAssiz, 1846b:246 (nomem nudum).

Navosoma BlanchaRD, 1846:206; THOMSON, 1860:313; 1864:297,

477; LACORDAire, 1869:94; CHENU, 1870:307; LAMEERE, 1901:322.

Naosoma AgAssiz, 1846b:245 (nomen nudum).

Naosoma SchjöDTE, 1864:497 (nomen nudum).

Naosoma SchJöDTE, 1865:194 (nomen nudum).

Naosoma Gemminger \& Harold, 1872:2763.

Naviosoma [sic]; BuRMEISTER, 1865:159.

Callipogon (Navosoma); LAMEere, 1904:67; 1913:46 (cat.); 1919:86; Melzer, 1919:95; BlaCKWELdER, 1946:553 (cat.); ZAJCIW \& Ruffinelli, 1962:16; Monné \& Giesbert, 1994:8 (cat.); Monné, 1995:19 (cat.); Monné, 2002:6 (hosp.).

Espécie-tipo. Navosoma triste Blanchard in D’Orbigny, 1846, monotipia (= Prionus luctuosus Schönherr, 1817).

BLANCHARD (1845: v) afirmou: "Les genres ayant une * sont caractérisés pour la première fois dans cet ouvrage". "Navosome" foi um dos gêneros descritos por BLANCHARD (1845), sem citação de nenhuma espécie, além do nome genérico estar grafado em vernáculo. Esses dois fatores impossibilitam a validação do gênero nessa data.

AgAssiz (1846a: 106) utilizou Navosoma como emenda para "Navosome" e atribuiu-o a BLANCHARD (1845). Esse ato nomenclatural não faz com que o nome genérico Navosoma seja validado em BLANCHARD (1845), porque é uma emenda para um nome inválido, portanto, igualmente um nomen nudum.

Agassiz (1846b: 246) escreveu: "Navosoma Blanch. Col. 1845. (Scr. Naosoma)". Na página anterior, AGASSIZ (1846b: 245) registrou: "Naosoma - ( $V$. Navosoma Blanch.) Col.". Essa nova emenda (para Navosoma) também é um nomen nudum.

SCHJöDTE $(1864,1865)$, aparentemente seguindo Agassiz (1846b), utilizou o nome genérico Naosoma. Apesar das emendas serem posteriores a BLANCHARD (1846) (vide discussão sobre $N$. triste) e portanto emendas injustificadas, consideremos Naosoma Schjödte, 1864 e 1865 como nomina nuda. ScHJöDTE (1864, 1865) não relacionou Naosoma com Navosoma Blanchard e não mencionou nenhuma espécie. Além disso, os caracteres genéricos mencionados nesses trabalhos, não permitem concluir com segurança, que os dois nomes (Naosoma e Navosoma) referem-se ao mesmo gênero.

Gemminger \& Harold (1872: 2763) citaram Naosoma como emenda para Navosoma Blanchard. Naosoma Gemminger \& Harold, 1872 é uma emenda 
injustificada e, portanto, um nome válido e sinônimo de Navosoma (vide observação abaixo).

SHERborn (1922) citou: "Navosoma L. Agassiz, Nom. Zool. (Col.) 1845 - Col. [Navosome, Blanch. vernac.]" e "Navosoma E. Blanchard in d'Orbigny, Amér. Mérid. (Ins.) 1846, 206. - Col. [vernac. 1845]”. Embora SHERBorn (1922) tenha atribuído a autoria do gênero a Agassiz [1845 (sic)], NeAve (1940: 272), esclareceu: "Navosoma Agassiz 1845, Nom. Zool. (Col.) [n.n.]; Blanchard 1846, in d'Orbigny, Amér. Mérid. (Ins.), 206. - Col.”. A abreviação "n.n.”, de acordo com Neave (1940) significa nomen nudum. Dessa forma, NeAve (1940) atribuiu a autoria de Navosoma à Blanchard (1846). Além disso, de acordo com Bowley \& Sмiтh (1968), o ano de publicação do fascículo XI da obra de Louis Agassiz, mencionada por SHERBORN (1922) e NEAVE (1940), foi 1846.

LAMEERE (1904:67) considerou Navosoma como subgênero de Callipogon e atribuiu incorretamente a autoria para BLANCHARD (1845:141).

O Index Universalis de AgAssiz foi publicado em 1847, segundo Bowley \& SMith (1868) e KeVAN (1970), apesar de constar o ano de 1846 na obra. EvEnHuis (1997:50) considerou o ano de 1846 como correto: "Sherborn (in litt.) stated that the BMNH received a copy of this work on 29 December 1846". Não conseguimos estabelecer se a obra de AgAssiz (1846b) foi publicada antes ou depois da obra de BLANCHARD (1846). Se AgASSIZ (1846b) foi publicada primeiro, então Naosoma é um nomen nudum. Se foi publicada depois de Blanchard (1846), então a autoria do nome genérico Naosoma é de AGASSIZ (1846b) e não de Gemminger \& Harold (1872). Na lista bibliográfica, mantivemos a autoria de Naosoma para GEMminger \& HAROLD (1872) e nomen nudum em AgASSIZ (1846b).

Navosoma difere de Callipogon Audinet-Serville, 1832: cabeça, incluindo as mandíbulas, nitidamente mais curta do que o protórax; distância entre os lobos oculares inferiores aproximadamente igual ao dobro da largura de um lobo; carena ocular dos machos, larga e pouco distinta; mandíbulas mais curtas do que o escapo; élitros com carenas; último artículo dos tarsos mais curto do que os demais reunidos. Em Callipogon, a cabeça, incluindo as mandíbulas, é mais longa do que o protórax, a distância entre os lobos oculares inferiores é, no mínimo, quatro vezes maior que a largura de um lobo, a carena ocular dos machos é distinta e estreita, as mandíbulas são mais longas do que o escapo (principalmente nos machos), os élitros não possuem carenas e o último artículo dos tarsos é mais longo, ou subigual, aos demais reunidos.

\section{Navosoma luctuosum (Schönherr, 1817)}

\section{Prionus luctuosus SCHÖNHERR, 1817: 346.}

Callipogon (Navosoma) luctuosus; LAMEere, 1904:67; BRUCH, 1912:181 (cat.); Lameere, 1913:46 (cat.); 1919: 86; Melzer, 1919:96; ZIKÁN \& ZIKÁN, 1944:3 (distr.); BosQ, 1947:8 (distr.); Bose \& RufFinelli, 1951:3 (distr.); BaUCKe, 1955:79; BiEZANKO \& Bose, 1956:4 (distr., hosp.); ZaJCIw, 1972:51 (distr.); VianA, 1972:228 (distr.).

Callipogon luctuosum; Blackwelder, 1946:553 (cat.); Silva et al., 1968:410 (hosp.); MonNé, 2004:17, 18, 69, 72 (hosp.).
Callipogon luctuosus; Buck, 1959:579 (distr.); PinHeIro, 1962:251 (hosp.); PIZA, 1968:17.

Callipogon (Navosoma) luctuosum; MonnÉ \& ZAJCIW, 1972:51 (distr.); Penteado-Dias, 1982:219 (larva); 1984:225, 226 (morfologia); Monné \& Giesbert, 1994:8 (cat.); Monné, 1995:19 (cat.); 2002:6 (hosp.).

Ergates Huberti BuQuet, 1840:28.

Navosoma triste Blanchard in D'Orbigny, 1846:206; White, 1853:34; Thomson, 1864:298; Pelseneer, 1890: 176 (distr.).

Naviosoma triste; BURMEISTER, 1865:159.

Naosoma triste; Gemminger \& Harold, 1872:2764.

Navosoma Blanchardii Thomson, 1877:270; 1878:3; Lameere, 1883:8 (cat.).

LAMEERE (1904:67) colocou Navosoma triste na sinonímia de Callipogon (Navosoma) luctuosus (Schönherr, 1817), e escreveu: "Navosoma triste Blanch., Voy. D'Orb., 1843, p. 206, t. 20, fig. 5". A autoria e as datas foram mantidas em LAMEERE (1913:46), LAMEERE (1919:86), BLACKWELDER (1946:553) (nome da espécie grafado como tristis). Monné \& GiesBert (1994:8) e Monné (1995:19) consideraram "Blanchard, 1843: 206" a autoria e ano de publicação, tanto para o gênero como para a espécie. MonNé (2002) registrou, equivocadamente, Navosoma e $N$. triste como descritos por BlaNCHARD in D'Orbigny (1847).

A obra de D'Orbigny (Voyage dans l'Amérique Méridionale), de acordo com o British Museum (NaturaL HISTORY) (1904:603), foi publicada em partes, entre os anos de 1837 e 1843[46]. SHerborn \& WoOdward (1901), esclareceram que o volume 6 , parte 2 , foi publicado como segue: páginas 1-16, em 1837; páginas 17-56, em 1838; páginas $57-88$, em 1842; páginas 89-104, em 1844; páginas 105-222, em 1846. Ainda de acordo com SHERBORN \& WoODWARD (1901), as páginas 1-60, são de autoria de A. Brullé e, dessa última até a página 222, de E. Blanchard. ERICHSON $(1847: 73,125)$ mencionou 1846 como o ano de publicação de N. triste. SHERBORN \& WoODWARd (1901) não esclareceram em que data foi publicada a estampa número 20, onde há um desenho de $N$. triste.

\section{Spiloprionus Aurivillius, 1897 stat. nov.}

Spiloprionus Aurivillius, 1897:241; LAMEere, 1901:321 (nota), 323

Callipogon (Spiloprionus); LAMeERe, 1904:50; 1913:45 (cat.); 1919:84; BlackWelder, 1946:553 (cat.); Monné \& Giesbert, 1994:8 (cat.); MonNÉ, 1995:21 (cat.).

Espécie-Tipo: Spiloprionus sericiomaculatus Aurivillius, 1897, monotipia.

Spiloprionus sericiomaculatus AuRIVILLIUS, 1897:242.

Callipogon (Spiloprionus) sericiomaculatus; LAMEERE, 1904:53; 1913:45 (cat.); 1919:84; MonNÉ \& Giesbert, 1994:8 (cat.); MonNÉ, 1995:21 (cat.).

Callipogon sericiomaculatus; LAMEERE, 1912:165

Callipogon sericiomaculatum; BLACKWELDER, 1946:553 (cat.).

LAMEERE (1904:50) considerou Spiloprionus como subgênero de Callipogon e comentou: "Il n'est pas possible, en effet, que dans cette espèce il n'y ait pas des mandibules très allongés chez le mâle major: si les mandibulbes du mâle étaient raccourcies, il y aurait une autre forme de dimorphisme sexuel chez le mâle...". Posteriormente, LAMEERE (1912:165) afirmou ter examinado 
dois machos de Callipogon sericiomaculatus no British Museum, mas não teceu nenhum comentário sobre as mandíbulas.

Spiloprionus difere de Callipogon por apresentar: mandíbulas curtas nos dois sexos; pronoto sem dimorfismo sexual; margens laterais do pronoto com três espinhos nítidos; escutelo dividido por carena longitudinal; tarsômeros mais estreitos. Em Callipogon, as mandíbulas dos machos de grande porte são longas, o pronoto apresenta dimorfismo sexual, os espinhos laterais do pronoto são curtos, o escutelo não é dividido por carena longitudinal e os tarsômeros são mais largos.

Agradecimentos. A Ziro Komiya e Hisayasu Arai, pela doação de parte dos espécimes utilizados neste trabalho. Ao Dr. Steven W. Lingafelter (USNM), pela execução das fotografias de Mallodon baroni Casey, 1912. Ao Dr. Carlos José Einicker Lamas (MZSP) e Daniele de Araújo Lopes (MNRJ), pelas informações extraídas da obra de Evenhuis (1997). A dois consultores anônimos, pelas valiosas correções e sugestões.

\section{REFERÊNCIAS BIBLIOGRÁFICAS}

AgAssiz, L. 1846a. Nomina systematica generum coleopterorum, tam viventium quam fossilum, secundum ordinem alphabeticum disposita, adjectis auctoribus, libris in quibus reperiuntur, anno editions, etymologia et familiis ad quas pertinent. Fascículo XI. In: Agassiz, L. Nomenclator Zoologicus continens nomina systematica generum animalium tam viventium quam fossilium, secundum ordinem alphabeticum disposita, adjectis auctoribus, libris, in quibus reperiuntur, anno editionis, etymologia et familiis, ad quas pertinent, in singulis classibus. Soloduri [= Solothurn, Suiça], Jent et Gassmann. xi + 170 p. 1846b. Nomenclatoris Zoologici. Index universalis, continens nomina systematica classium, ordinum, familiarum et generum animalium omnium, tam viventium quam fossilium, secundum ordinem alphabeticum unicum disposita, adjectis homonymiis plantarum, nec non variis adnotationibus et emendationibus. In: Agassiz, L. Nomenclator Zoologicus continens nomina systematica generum animalium tam viventium quam fossilium, secundum ordinem alphabeticum disposita, adjectis auctoribus, libris, in quibus reperiuntur, anno editionis, etymologia et familiis, ad quas pertinent, in singulis classibus. Soloduri $[=$ Solothurn, Suiça $]$, Jent et Gassmann. viii + 393 p.

Aurivillius, C. 1897. Neue oder wenig bekannte Coleoptera Longicornia. 5. Entomologisk Tidskrift 18:241-248.

Baucke, O. 1955. Catálogo dos insetos encontrados no Rio Grande do Sul, Col., Cerambycidae. Boletim da Secretaria da Agricultura, Indústria e Comércio do Rio Grande do Sul 1:1-87.

Biezanko, C. M. \& Bosq, J. M. 1956. Cerambycidae de Pelotas e seus arredores. Agros 10(3-4):3-15.

Blackwelder, R. E. 1946. Checklist of the coleopterous insects of Mexico, Central America, the West Indies and South America. Part 4. Bulletin of the United States National Museum 185:551-763.

BlanCHARD, C. E. 1845. Histoire des Insectes, traitant de leurs moeurs et de leurs métamorphoses en général, et comprenant une nouvelle classification fondée sur leurs rapports naturels. Paris, Didot. v.2, p.1-524.

1846. Insectes de L'Amérique méridionale, recueillis par Alcide d'Orbigny. In: D'OrbignY, A. Voyage dans l'Amérique Méridionale (Le Brésil, la République Orientale de l'Uruguay, la République Argentine, la Patagonie, la République du Chili, la République de Bolivia, la République du Pérou), exécute pendant les années $1826,1827,1828,1829,1830,1831,1832$ et 1833, par Alcide d'Orbigny. Paris, P. Bertrand. v.6, part2, p.61-222.

BosQ, J. M. 1947. Catálogo preliminario de los Coleópteros del Paraguay, parte 3. Revista de la Sociedad Científica del
Paraguay 7(2):5-32.

Bose J. M. \& Ruffinelli, A. 1951. Notas para el catálogo de los Cerambícidos del Uruguay. Comunicaciones Zoológicas del Museo de Historia Natural 3(62):1-32.

Bowley, D. R. \& Smith, H. M. 1968. The dates of publication of Louís Agassiz's Nomenclator Zoologicus. Journal of the Society for the Bibliography of Natural History 5(1):35-36.

British Museum (Natural History). 1904. Catalogue of the Library of the British Museum (Natural History). London, British Museum (Natural History). v.2, p.501-1038.

Browne, J. \& Peck, S. B. 1996. The long-horned beetles of south Florida (Cerambycidae: Coleoptera): biogeography and relationships with the Bahama Islands and Cuba. Canadian Journal of Zoology 74(12):2154-2169.

Bruch, C. 1912. Catálogo sistemático de los Coleópteros de la República Argentina. Pars VIII. Familia Cerambycidae (Prionini, Cerambycini, Lamiini). Revista del Museo de La Plata 18:179-226.

Buck, P. 1959. Cerambycidae in der Sammlung des Instituto Anchietano de Pesquisas. Pesquisas 3:577-609.

Buquet, J. B. 1840. Un longicorne du Brésil, de la tribu des prioniens et du genre Ergates. Bulletin de la Société Entomologique de France 1840:27-28.

Burmeister, H. 1865. Longicornia Argentina. Systematische Üebersicht der Bockkäfer der La Plat-Staaten. Stettiner Entomologische Zeitung 26:156-181.

Calle.com. 2004. Global gazetteer version 2.1. Disponível em: <http://www.fallingrain.com/world/>. Acesso em: 03.2005 .

Casey, T. L. 1912. Studies in the Longicornia of North America. Memoirs on the Coleoptera 3:215-376.

CHEMsAк, J. A. 1996. Illustrated revision of the Cerambycidae of North America. I. Parandrinae, Spondylidinae, Aseminae, Prioninae. Burbank, Wolfsgarden. $\mathrm{x}+149 \mathrm{p}$.

Chemsak, J. A. \& Linsley, E. G. 1982. Checklist of Cerambycidae and Disteniidae of North America, Central America, and the West Indies (Coleoptera). Medford, Plexus. 138 p.

Chemsak, J. A. \& Noguera, F. A. 1993. Annotated checklist of the Cerambycidae of the Estacion de Biologia Chamela, Jalisco, Mexico (Coleoptera), with descriptions of new genera and species. Folia Entomologica Mexicana 89:55-102.

Chemsak, J. A.; Linsley, E. G. \& Nogueira, F. A. 1992. Listados faunísticos de México. II. Los Cerambycidae y Disteniidae de Norteamérica, Centroamérica y las Indias Ocidentales (Coleoptera). México, D. F., Universidad Nacional Autónoma de México. 204p.

Chenu, J. C. 1870. Encyclopédie d'histoire naturelle. Coléoptères. Paris, Marescq \& Compagnie. v. 3, vi + 360p., 296 figs, 48 pls.

Erichson, W. F. 1847. Bericht über die wissenschaftlichen Leistungen in der Naturgeschichte der Insecten, Arachniden, Crustaceen und Entomostraceen während des Jahres 1846. Archiv für Naturgeschichte 1847(2):65-208.

Evenhuis, N. L. 1997. Litteratura Taxonomica Dipterorum (1758-1930). Being a selected list of the books and prints of Diptera taxonomy from the beginning of Linnaean zoological nomenclature to the end of the year 1930; containing information on the biographies, bibliographies, types, collections, and patronymic genera of the authors listed in this work; including detailed information on publication dates, original and subsequent editions, and other ancillary data concerning the publications listed herein. Leiden, Backhuys. v.1, 426p.

Fragoso, S. A. \& Monné, M. A. 1995. Notes on Macrotomini (Coleoptera, Cerambycidae, Prioninae). Revista Brasileira de Biologia 55(2):215-225.

Gemminger, M. \& Harold, E. 1872. Catalogus Coleopterorum hucusque descriptorum synonymicus et systematicus. Monachii (= Mônaco), Sumptu E. H. Gummi. v.9, p.26692988. 
Hovore, F. T. \& Santos-Silva, A. 2004. Notes and descriptions of Neotropical Macrotomini (Coleoptera, Cerambycidae, Prioninae). Revista Brasileira de Entomologia 48(1):49-54

Kevan, D. K. M. 1970. Agassiz's Nomenclatoris Zoologici Index Universalis - a correction. Journal of the Society for the Bibliography of Natural History 5(4):286.

Lackerbeck, K. 1998. Neue und wenig bekannte Prioninae (Coleoptera, Cerambycidae). Entomofauna Zeitschrift für Entomologie 19(32):517-524

Lacordaire, J. T. 1869. Histoire Naturelle des Insectes. Genera des Coléoptères ou exposé méthodique et critique de tous les genres proposés jusqu'ici dans cet ordre d'insectes. Paris, Librairie Encyclopédique de Roret. v.9, n.1, p.1-409.

Lameere, A. 1883. Liste des Cérambycides décrits postérieuremen au catalogue de Munich. Annales de la Société Entomologique de Belgique 26:1-78.

1901. Étude sur la phylogénie des longicornes. Annales de la Société Entomologique de Belgique 45:314-323.

1904. Révision des Prionides. Neuvième mémoire Callipogonines. Annales de la Société Entomologique de Belgique 48:7-78.

1912. Révision des Prionides. Vingt-Deuxième mémoire Addenda et corrigenda. Mémoires de la Société Entomologique de Belgique 21:113-188.

1913. Coleopterorum Catalogus, pars 52, Cerambycidae. Prioninae. Berlin, W. Junk. 108p.

1919. Genera Insectorum. Coleoptera, Fam. Cerambycidae, subfam. Prioninae. Bruxelles, P. Wytsman. v. 172, 189 p.

LeConte, J. L. 1851. An attempt to classify the longicorn Coleoptera of the part of America, north of Mexico. Journal of the Academy of Natural Sciences of Philadelphia 2(2):99-112.

Linsley, E. G. 1957. Descriptive and synonymical notes on some North American Cerambycidae (Col.). American Museum Novitates 1828:1-21.

Linsley, E. G. \& Chemsak, J. A. 1997. The Cerambycidae of North America . Part VIII. Bibliography, Index and Host Plant Index. University of California Publications in Entomology 117:1-534.

Melzer, J. 1919. Os longicórnios brazileiros da subfamilia "Prioninae", tomando em consideração particular as espécies do Estado de São Paulo. Revista do Museu Paulista 11:1-207.

Monné, M. A. 1995. Catalogue of the Cerambycidae (Coleoptera) of the Western Hemisphere. Part XXII. São Paulo, Sociedade Brasileira de Entomologia. 115p.

2002. Catalogue of the Neotropical Cerambycidae (Coleoptera) with known host plant - part V: Subfamilies Prioninae, Parandrinae, Oxypeltinae, Anoplodermatinae, Aseminae and Lepturinae. Publicações Avulsas do Museu Nacional 96:1-72.

2004. Catalogue of the Neotropical Cerambycidae (Coleoptera) with known host plant - part VI: host plant index. Publicações avulsas do Museu Nacional 100:1-95.

Monné, M. A. \& Giesbert, E. F. 1994. Checklist of the Cerambycidae and Disteniidae (Coleoptera) of the Western Hemisphere. Burbank, Wolfsgarden. 410p.

Monné, M. A. \& ZaJciw, D. 1972. Cerambícidos del Uruguay, nuevos o poco conocidos. III. Atas da Sociedade de Biologia 15(2):49-53.

Neave, S. A. 1940. Nomenclator Zoologicus. A list of the names of genera and subgenera in zoology from the tenth edition of Linnaeus 1758 to the end of 1935. London, The Zoological Society of London. v.3, 1065p.

Noguera, F. A. \& Chemsak, J. A. 1996. In: Bousquets, J. L.; García-Aldrete, A. N. \& GonZÁlez-Soriano, E. Biodiversidad, taxonomía y biogeografía de artrópodos de México: hacia una síntesis de su conocimiento. México, Universidad Nacional Autónoma de México. p.381-409.

PelseneER, P. 1890. Première note sur les coléoptères recueillis par M. Ed. Van Beneden dans l'Amérique méridionale. Comptes-Rendus des Séances de la Société Entomologique de Belgique 11(4):174-176.
Penteado-Dias, A. M. 1982. Callipogon (Navosoma) luctuosum (Schoenherr, 1817): notas sobre a biologia, descrições da larva e pupa (Coleoptera, Cerambycidae) Revista Brasileira de Entomologia 26(3/4):219-224

1984. Estudo comparativo do cordão nervoso nos Cerambycidae (Coleoptera). Revista Brasileira de Entomologia 28(3):223-243.

Pinheiro, J. V. 1962. Contribuição para o conhecimento de insetos dos eucaliptais no Brasil. Anuário Brasileiro de Economia Florestal 14(14):245-255.

Piza, S. T., JR. 1968. Insetos de Piracicaba. São Paulo, Edição comemorativa. Bicentenário de Piracicaba. Escola Superior de Agricultura "Luiz de Queiroz". 123p.

Santos-Silva, A. \& Martins, U. R. 2003. Revisão do gênero Physopleurus Lacordaire, 1869 e notas em Macrotomini (Coleoptera, Cerambycidae, Prioninae). Revista Brasileira de Entomologia 47(2):245-265.

SAY, T. 1824. Descriptions of Coleopterous Insects collected in the late Expedition to the Rocky Mountains, performed by order of Mr. Calhoun, Secretary of War, under the command of Major Long. Journal of the Academy of Natural Sciences of Philadelphia 3(2):298-331.

SchjöDte, J. C. 1864. Danmarks cerambyces. Naturhistorisk Tidsskrift 3(2):483-576.

1865. On the classification of Cerambyces, with particular regard to the Danish Fauna. The Annals and Magazine of Natural History 15(Serie 3):182-209.

SCHÖNHERR, C. J. 1817. Synonymia Insectorum, oder: Versuch einer Synonymie aller bisher bekannten Insecten, nach Fabricii Systema Eleutheratorum \& c. geordnet. Skara, Lewerentzischen Buchdruekerey. v.1, part $3,506 \mathrm{p}$.

Sherborn, C. D. 1922. Index Animalium. Sive index nominum quae ab A.D. MDCCLVIII. Generibus et speciebus animalium imposita sunt. Sectio Secunda. A Kalendis ianuariis, MDCCCI usque ad finem decembris, MDCCCL. London, British Museum (Natural History). v. 4, p. 3747-4450.

Sherborn, C. D. \& Woodward, B. B. 1901. Notes on the Dates of Publication of the Natural History portions of some French Voyages - Part I. 'Amérique méridionale'; 'Indes orientales'; "Pôle Sud' ('Astrolabe' and 'Zélée'); 'La Bonite'; 'La Coquille'; and 'L'Uranie et Physicienne'. The Annals and Magazine of Natural History 7(Serie 7):388-392

Silva, A. G. A.; Gonçalves, C. R.; Galvão, D. M.; Gonçalves, A. J. L.; Gomes, J.; Silva, M. N. \& Simoni, L. 1968. Quarto catálogo dos insectos que vivem nas plantas do Brasil. Seus parasitos e predatores. Rio de Janeiro, Ministério da Agricultura. v.1, n.2, p.1-622

Тномson, J. 1860. Essai d'une classification de la famille des cérambycides et matériaux pour servir à unemonographie de cette famille. Paris. $404 \mathrm{p}$.

1864. Systema cerambycidarum ou exposé de tous les genres compris dans la famille des cérambycides et familles limitrophes. Mémoires de la Société Royale des Sciences de Liège 19:1-540.

1867. Révision du groupe des Mallodonites (insectes, coleóptères, prionites, cérambycides). Recueil d'Histoire Naturelle 1(2):85-106.

1877. Typi cerambycidarum Musei Thomsoniani. Revue et Magasin de Zoologie (3) 5:249-279.

1878. Typi cerambycidarum musei Thomsoniani Paris, E. Deyrolle. 21p.

Toledo, V. H.; Noguera, F. A.; Chemsak, J. A.; Hovore, F. T. \& Giesbert, E. F. 2002. The Cerambycid fauna of the tropical dry forest of "El Aguacero," Chiapas, Mexico (Coleoptera: Cerambycidae). The Coleopterists Bulletin 56(4):515-532.

VianA, M. J. 1972. Aporte al Catálogo de Cerambycidae del Paraguay (Insecta, Coleoptera). Revista del Museo Argentino de Ciencias Naturales "Bernardino Rivadavia" (Entomología) 3(4):207-405.

Villiers, A. 1980. Coléoptères Cerambycidae des Antilles Françaises. I. Parandrinae, Prioninae, Lepturinae. Annales 
de la Société entomologique de France, (N. S.) 16(1): 133-157.

White, A. 1853. Catalogue of the coleopterous insects in the collection of the British Museum. Longicornia I. London, Brithish Museum (Natural History) v.7, p.1-174.

YANEGA, D. 1996. Field guide to Northeastern longhorned beetles (Coleoptera: Cerambycidae). Champaign, Illinois Natural History Survey. 174p. (Manual, 6).
Zajciw, D. 1972. Contribuição para o estudo da fauna dos longicórneos do Parque Nacional do Itatiaia (Coleoptera, Cerambycidae). Brasil Florestal 3:40-72.

Zajciw, D. \& Ruffinelli, A. 1962. Fauna de los Cerambícidos del Uruguay. Boletín de la Facultad de Agronomia 60:1-89.

ZIKÁN, J. F. \& ZiKÁN, W. 1944. A inseto-fauna do Itatiaia e da Mantiqueira. Boletim do Ministério da Agricultura 33(8): 1-50.

Recebido em março de 2005. Aceito em setembro de 2005. ISSN 0073-4721

Artigo disponível em: www.scielo.br/isz 